\title{
"I Feel Healthy Like Any Other Person" Reasons for Defaulting from ART Treatments Use Among Adults Living with HIV in Tunduru District: A Qualitative Inquiry
}

\author{
Emmy Metta ${ }^{1, *}$, William Yonathan ${ }^{1,2}$, Melkizedeck Leshabari $^{1}$ \\ ${ }^{1}$ School of Public Health and Social Sciences, Muhimbili University of Health and Allied Science, Dar es Salaam, Tanzania \\ ${ }^{2}$ Tanzania Health Promotion Support, Dar es Salaam, Tanzania
}

Email address:

emetta2000@gmail.com (E. Metta)

${ }^{*}$ Corresponding author

\section{To cite this article:}

Emmy Metta, William Yonathan, Melkizedeck Leshabari. "I Feel Healthy Like Any Other Person” Reasons for Defaulting from ART Treatments Use Among Adults Living with HIV in Tunduru District: A Qualitative Inquiry. International Journal of HIV/AIDS Prevention, Education and Behavioural Science. Vol. 7, No. 1, 2021, pp. 8-14. doi: 10.11648/j.ijhpebs.20210701.12

Received: March 1, 2021; Accepted: March 12, 2021; Published: March 22, 2021

\begin{abstract}
Background: Continuing use of ART treatments is reported to improve health and health outcomes of people living with HIV. However, adherence to ART prescriptions is generally a challenge and many people default from HIV care and treatment. This study assessed reasons for ART treatment default among adults living with HIV in Tunduru district Tanzania. Methods: We conducted a qualitative cross-sectional study between January and February 2018. Ten key informants who had defaulted from HIV care and treatment for five or more months were engaged in in-depth interviews on reasons behind defaulting. Thematic analysis using NVivo was conducted to identify emerging themes. Results: Reasons for defaulting from ART treatments which emerged include being tired of using the ART medications for a long time, not experiencing HIV related symptoms, fear of stigma and shame of being known to use ART, inadequate food, fear of side effects and use of local herbs as alternative. Other reasons include long distance to the health facilities and long waiting time for the services at the facility. Conclusion: Reasons for defaulting from HIV treatment and care among adults are multifaceted in nature requiring multifaceted efforts to address them if the gains intended from the use of ART are to be achieved and sustained.
\end{abstract}

Keywords: ART, Default, Non-adherence, Tunduru, Tanzania

\section{Introduction}

Several strategies to control the HIV pandemic have been proposed by the World Health Organization and adopted by member countries including Tanzania. Among them is the 9595-95 ambitious treatment goal which aims to end the epidemic. It is proposed that $95 \%$ of people living with HIV should know their HIV status, and $95 \%$ of those diagnosed should receive sustained antiretroviral therapy and 95\% of those on treatment should attain viral suppression by the year 2030 [1]. Attainment of this goal assumes that people diagnosed with HIV infection will adhere to the advice and medications prescribed by health care providers including going for scheduled clinic appointments. However, defaulting from treatment is reported to be common among those who are on ART treatments.
Poor patient adherence to prescribed ART regimen has negative implications not only to patients themselves but also to health care services and communities in general. ART non adherence is associated with poor treatment outcomes, development of viral resistance, increased risk of disease progression and the need to use expensive second- or thirdline ART regimens, increased healthcare expenditure as well as avoidable deaths [2]. ART non-adherence also increases the risk of disease progression to AIDS, developing opportunistic infections and increased chances of infecting other people in communities [3-5].

ART adherence requires PLWHIV to stick firmly to the treatment regimen by taking the right medicine, the right dose, at the right time, at the right frequency, as prescribed by health care providers $[3,6]$. However, sustained adherence to recommended care and treatment is generally a challenge in 
chronic disease management $[7,8]$ and in HIV/ AIDS in particular [9]. This has been observed in different settings [10-12]. Since 2004 the Tanzanian government through the National AIDS Control Program has been providing antiretroviral therapy (ART) to individuals living with HIV. The service is provided free of charge in all public health facilities. Services provided include antiretroviral therapy, counseling on adherence to ARV, treatment of opportunistic infection, nutrition counseling and growth monitoring. By 2019 about $75 \%$ of people living with HIV in Tanzania were on ART treatment and among them, 69\% were virtually suppressed [13]. It has been pointed out that to sustain the gains achieved, there is a need to ensure individuals infected with HIV adheres to their prescribed treatment since ART is only effective if patients adhere to the prescribed regimens [14]. This paper is based on qualitative information which was obtained from clients who defaulted from ART treatments for five or more consecutive months in one of the rural districts in southern Tanzania.

\section{Methodology}

The study was conducted in Tunduru, a rural district in Ruvuma Region Southern Tanzania between January and February, 2018. The district has a total population of 298,279 including 143660 (males) and 154619 (females). HIV prevalence in Tunduru district is $3.2 \%$ among the adult population and is one of the districts in Tanzania that has a large number of people defaulting from their ART use. By the end of 2017; a total of 29,544 people living with HIV (PLWHIV) in the district was registered in care and treatment clinics and 20.7\% were ART defaulters (Tunduru District Health Report, 2017). The reasons for defaulting from ART treatments in this rural setting were not clear.

The district has 13 health facilities and all offers free of charge CTC services including ART to PLWHIV. Five of these health facilities that had the highest number of PLWHIV were selected as study sites. In each of the facilities document review was conducted to identify a list of people who had been on ART treatments for more than three years and stopped clinic attendance. From the list those who had missed clinic appointments for five or more months were identified. Finally, two people with the highest number of missed clinic appointments (per health facility) were purposively identified and recruited for the study. Ethical clearance to conduct the study was obtained from Muhimbili University of Health and Allied Sciences (MUHAS) Ethical Review Board while permission to access the key informants was obtained from Ruvuma Region and Tunduru District administrative authorities.

The research team contacted each of the individuals identified and scheduled appointments with them at their preferred venue. They mainly agreed to be visited at home. Interviewers visited them at home and introduced about the study, each key informant was informed in detail about the study, given time to ask questions and asked about the willingness to participate. After expressing willingness to be part of the study a written informed consent was obtained.

Data was collected using in-depth interviews (IDI) guides. A team of two social scientists experienced in conducting health related qualitative studies conducted in-depth interviews. The themes covered include: perceptions of HIV and its treatments, experiences with the use of ART, source of HIV related services including ART, and the reasons for missing clinic appointments. The interviews which lasted between 40 and 50 minutes were conducted in Swahili, a Tanzanian national language which is spoken by most people in the country. All interviews were audio recorded and verbatim transcriptions were made within 24 hours from the time they were conducted. A total of 10 in-depth interviews were conducted, however, data saturation was reached at the eight's interview and yet two more interviews were added to confirm saturation.

Interview transcripts were reviewed and cross checked against the audio files for quality by the first and the second authors before they were imported into NVivo 9 (QSR International Pty Ltd, Australia). Thematic analysis was used to analyze the data following five stages as described by Braun and Clarke [15] to establish meaningful patterns in the data: familiarization with the data, generating initial codes, searching for themes among codes, reviewing themes and presenting the results. The coding also involved identification of the typical quotes that are presented to illustrate the various themes of interest.

\section{Results}

The study participants consisted of five men and five women. Their age ranged from 32 to 55 years with a median of 42 years and all had primary school education. Only one was single and the rest were married. In terms of occupation, they were either peasant farmers (5) or self-employed (5). The following were the major reasons for defaulting from ART treatment:

1. Exhaustion from long time medication use

2. Lack of HIV related symptoms

3. Fear of stigma and embarrassment

4. Distance to the health facility

5. Inadequate food

6. Fear of side effects

7. Use of local herbs

8. Long waiting time at the facility

\subsection{Exhaustion from Long Time Medication Use}

Some of study participants expressed that the nonadherence to ART medications was due to exhaustion arising from use of ART treatment for a long time. They reported to have initially used their prescribed medications as instructed but later became tired and lazy of continuing to use them. All study interviewed participants voiced concerns that using medicines for a long time is tiresome and difficult to follow because there are always temptations to stop the medicines. They spoke about non-adherence to have started by skipping doses when they felt healthy, a behavior that continued until 
one decided to stop using the treatments. A woman aged 35 years said:

"At the beginning I used them (ART) very well...I did not skip my dose even for a single day for about two years.... thereafter, I became really tired and lazy about taking them every day, so you know...I would skip some days when I felt healthy and continue with my usual life .... it continued like that when my body seemed not well, I took them again and when felt healthy I skip and after sometimes I did not feel anything different in my body ...I am well ... I feel healthy like any other person.... I do not use them anymore" (Female, 35 years).

When explaining the difficulty of daily medication use and required adherence another participant said:

"I got tired of using them daily... it really made me tired and uncomfortable,...you know taking medicines every day is not a simple task.... it needs one to have a great heart ... always you feel like let me skip today.... I will use it tomorrow, for sure it is tiresome" (Male 33 years).

\subsection{Lack of HIV Related Illness Symptoms}

Some participants reported to have stopped using ART treatment because they experienced no pain or illness symptom.

"I did not experience any pain in my body or any illness symptom related to HIV" (Male 43 years).

Not feeling any HIV related illness symptoms were common expressions which emerged. One of the participants questioned the logic for using medicines daily while not feeling sick saying that:

"I was told that I have HIV and should use ART treatments daily for the rest of my life...but I am not feeling sick...there is no pain anywhere in my body... I do not have any HIV related symptom ... the issue disturbing me is why I should continue using the medicines everyday while I am not feeling any sicker" (Female, 55 years).

\subsection{Fear of Stigma and Embarrassment}

Fear of stigma and embarrassment were among reasons why use of ART was stopped. All study participants had a potential fear for their HIV status to be known in the community. To ensure confidentiality of their HIV status they used prescribed medications in areas they believed no one can find out. Challenges of getting such venues contributed to inconsistent use ART as prescribed. None of the participant had revealed their HIV status to their relatives, neighbors, friends or peers. They said they did not want anyone to know their HIV status or use of ART due to fear of gossip of their condition by other people.

"None of my relatives know that I am HIV positive.... I never revealed it to anybody...I never took the medicines when other people were looking at me or were around...I always had to hide while taking them because I was afraid if people see me taking ART they would start murmuring about me all the time" (Female, 32yrs).

Due to perceived stigma, some informants said they registered for ART refills at CTC clinics which were far from their home villages because they feared being seen visiting the facility raised the possibility of being suspected to be on ART. In their villages, they said, if people see you frequently going to the CTC clinic, they will start pointing fingers at you as being HIV positive and that could be the beginning of stigma, contempt and discrimination.

In the study area many families and people live in close contacts with extended families and neighbors. Keeping one's health status secret was quite a challenge. It was noted that there was a close interaction between the key informants and members of their family including support and escort to clinic when one was not feeling well. Consequently, participants said that monthly CTC clinic visits to refill their ART medicines and taking them at prescribed times and doses was unbearable challenge. They said they experienced difficulties not only in concealing to other people that they were on ART but also in hiding the prescribed medications to ensure were not seen by anyone:

"You know there is always someone around at home.... I needed to be very smart to not let anyone notices if something is ongoing...I sometimes used the medicines in the kitchen or bathrooms.... I did not want anyone to know I am using medicines.... I made a hole in the kitchen and kept all my medicines there... I kept them there throughout and used them when I was sure nobody could see me" (Female, 41years).

\subsection{Distance to Health Facilities}

Distance from home to the CTC clinics and the associated transportation costs were reported as barriers to accessing ART treatment refills. None of the participants reported to stay within a walking distance to a health facility they registered for care. Some reported to travel more than $5 \mathrm{kms}$ to CTC clinic when they wanted to refill their ART medications. It was reported that ART use continuity was compromised by transport barriers especially fare to and from the CTC clinic for refill. Lack of money for transport to and from clinics was mentioned as a contributory factor to failure to attend clinic appointments and continue using ART. One of the participants when expressing why she missed her CTC clinic appointments and currently not using ART said:

"... I most of the time failed to go to refill my medicines because I did not have money to pay for the transport fare ...that is why I skipped many of my appointments......I have not used ART for quite some times now..." (Female, 41years)

Lack of money for the transport costs to the clinic was a common response from the study participants:

“.... I had to travel many kilometers to the health facility.... this required me to ensure that I had enough to pay for transport something that was not easy.... it is long now I could not attend my CTC dates...nothing I can do ....... I don't have money ...my income is not reliable..." (Male 52years)

Study participants complained that apart from the transport costs to the CTC clinic, one had to buy food while waiting 
for services at the clinics. This is because, they said, it takes longer to reach the health facility and by the time one gets there found many people waiting for service.

“... It takes too long for the bus to reach there ... (clinic) and there you found already many people are queuing to be attended..... So I used to stay too long in a queue waiting to be attended while already feeling hungry...this actually forces me to incur additional costs to buy food..." (Female 43 years)

\subsection{Inadequate Food}

Participants also talked about inadequate food as among the reasons for poor ART adherence. Key informants pointed out that the prescribed medicines were strong and made one feel bad when taken on an empty stomach. To feel better, they said, an HIV positive person is supposed to have eaten a sufficient amount of food before he/she uses the ART drugs. Some said they had skipped using their ART treatments due to lack of adequate food to eat. Others claimed that ART made them hungry all the time and increased their appetite.

"ART medicines increase appetite, they made me feel hungry all the time.... even if I had eaten enough food before using the medicines, I still felt like wanting to eat again and again .... I can say my appetite doubled...I was in trouble because I did not have enough food to eat all the time... $i$ had to stop using them ...I know my situation .... and I don't have money to guarantee enough eating to be able to use them" (Female, 32years).

Study participants talked about challenges of feeding themselves and their families and rarely managed to eat three meals a day. They said that one meal a day was not enough for the body to generate the required energy to accommodate ART drugs. One participant said that taking ART medicines on an empty stomach should be avoided, as she once experienced severe stomach pains due to that.

Furthermore, a concern was raised on the quality of food they ate and the limited choices of the variety of foods available as a challenge to their adherence to ART prescriptions:

"Many times I eat what is available ...there is no choice, quality is not a priority...... if it is my luck day I eat breakfast, lunch and dinner but these are rare occasions...in a normal routine I eat breakfast, and supper there is no lunch ..... This is not to say that the food is of good quality but just that I ate something ..... And because of not eating well, I became weak and had to make a decision of ending use of the ART medicines..." (Male 43 years).

\subsection{Fear of Side Effects}

Some participants said they stopped using ART due to fear of side effects. Among the side effects mentioned include: increased hunger, nausea, vomiting, diarrhea, fatigue and rashes all over the body. Others reported experiencing anxiety and difficult sleeping:

"I developed rashes, difficult sleeping, severe headache and nightmare. I felt as if I was about to die.... the only thing was just to stop taking ART...two of my friends also had the same experience" (Female, 41years).

Other participants associated experienced side effects with taking ART on an empty stomach.

"I tell you if you take it on empty stomach you real feel bad, it pains a lot, I used to sweat and tremble, felt dizzy, felt like my heart had been misplaced, felt very uncomfortable.... I mean you feel that bad hunger which you cannot even walk, seat or sleep, I was getting that kind of un comfort ...it is when I decided to stop using those medicines" (Female 43 years).

Some key informants said the side effects sometimes disappeared when they stopped using the ART drugs. They also said other conditions such as skin rashes and headache took time to disappear.

\subsection{Use of Local Herbs}

Local herbs were also used as part of treatment together with ART among the key informants. All of them said they had used local herbs at least once after being diagnosed with HIV. They said the used local herbs was for treating opportunistic infections such as diarrheas, stomach pain, skin rashes and itching, flue, fungus, coughing and chest pain. It was pointed out that previously people diagnosed with HIV were given free treatment for opportunistic infections at CTC clinics. At the time this study was conducted HIV positive patients diagnosed with other conditions were sent to the outpatient department for consultation and treatment. At the outpatient department, one was required to pay for all services and when she/he cannot afford the costs she/he was not attended. At times, they said, one may be able to pay $t$ for consultations and laboratory tests and thereafter be given a prescription to purchase prescribed medicines from private facilities.

"Previously if diagnosed with other conditions such as typhoid or pneumonia treatment was free of charge...but these days we go to the outpatient department...there treatments are not free, you are asked to pay just like any other person...even when there are no medicines they still ask you to pay to see the doctor and for laboratory tests.... at the end you are given a piece of paper and be instructed to buy the written medicines private drug stores... this is a big challenge we are experiencing.... where will you get the money?... when you don't have money to pay many of us resort to using local herbs.... when you use the herbs, you cannot mix with ART ...you may poison yourself ...." (Male 51years).

It was further pointed out that PLWHIV frequently get opportunistic infections and were required to pay for treatments from their own sources each time they got sick. This contributed to decisions to use of local herbs which were sometimes believed to be better than ART prescriptions.

"We people living with HIV get infections frequently and when thinking of the treatment costs at the health facilities..... Mmh some of us decided to use local herbs... and some of the local herbs have the ability to treat the infection and control the virus... you feel well sometime feeling better than the person on ART" (Male 43years). 


\section{Discussion}

This study has revealed a variety of contextual factors which contribute to reasons why people in rural settings default from HIV treatment. In order for the goal to end the HIV epidemic to be met, there is need for interventions which address challenges faced at individual, community and health system levels. Fear of side effects of the prescribed ART drugs contributed to defaulting from using prescribed ART drugs. The association between side effects and defaulting from ART treatments were also reported in Botswana [16] Demographic Republic of Congo [17] and Tanzania, Uganda and Zambia [18]. These findings suggest a need for continuous education on the potential negative reactions from ART treatments and counseling of PLWHIV on how to mitigate the reactions and the necessity to continue using their medications for improved treatment outcomes.

The fear of stigma and discrimination from revealing ones' HIV positive status contributed to secrecy on ART use which key informants said to some extent led to stopping using prescribed ART drugs. The finding is in line with what was reported from Malawi where denial of HIV positive status contributed to missed medications and defaulting from ART treatment [19]. Evidence on the role of disclosure of HIV positive status and improved ART adherence and patients' health in general is well documented [20]. HIV status disclosure is associated with increased social support, stronger family and relationship cohesion, reduced anxiety and depression, emotional as well as financial support [21, 22]. Interventions targeting ART adherence should address underlying causes of perceived stigma and discrimination associated with revealing the HIV status to significant others by those diagnosed to be HIV positive. The Tanzanian government through the country operational manual for comprehensive differentiated delivery of HIV and AIDS services outlines the need of all HIV positive individuals to have treatment supporters to encourage medication adherence [23]. However, it appears what policy documents propose what may not be happening in health facilities in rural settings.

ART treatment was free of charge when key informants first visited CTC clinics for diagnosis. However, access to the CTC clinics for the monthly follow-up and medical refills was reported to be challenging primarily because of the distance to the CTC clinics and the associated costs including transport and food when waiting to be attended at the health facility. Similar observations have also been reported from Malawi [24] and Kenya [25] where ART medical refills and adherence to ART treatments were hampered by the physical distance to health facilities and the associated costs. However, this partially may be due to choose of where to go for followup due fear of other people knowing the HIV status of those diagnosed with HIV. There is a need to address this challenge so that HIV positive individuals can be perceived by relatives and other community members just like other people with chronic diseases such as diabetes or hypertension There is also a need to identity a mechanism to address reasons contributing to treatment default that are outside patients control such as failing to pay for transport and meals. One option could be use of mobile services which reach clients at venues close to their homes.

Life-long medication and adherence to prescribed treatment regimens is a recommended best health practice to people with chronic illness conditions including those living with HIV, also a principal determinant to treatment success [26]. Failure to use recommended treatments is associated with reduced effectiveness of the treatments and increased morbidity, mortality and unnecessary health care costs [27, 28]. In this study some of the participants took deliberate actions to default ART treatment simply because they perceived to be healthy and did not experience any symptoms. Similar observations have been reported from studies done in Nigeria [29] and South Africa [30]. These findings are contrary to what was observed by Watt et al., [31] in other settings in Tanzania where improvement in health status motivated them to adhere to ART treatment. Interventions aiming at improving adherence to life-long medication should also emphasize to patients the health benefits of continuing to use prescribed medication even when they feel healthy or experience no symptoms.

Some key informants in this study believed that local herbs cure the HIV virus. Perceptions that local herbs can cure chronic diseases have also been reported from Ghana [28]. Use of local herbs to treat HIV/AIDS and related health challenges seem to be widespread in many African cultural settings [32-35]. While use of local herbs can be encouraged in situations where there is some evidence on their effectiveness, care should be taken to discourage using such herbs with prescribed medicines interchangeably due to potential risks of adverse interactions with the conventional medicines [36].

Similar to what was observed in the study, inadequate food was reported as an important aspect shaping HIV/AIDs patient decisions on adherence to care and ART treatments [37]. The study participant attributed inadequate food with increased risk of developing side effects hence avoided using ART when had inadequate food or on empty stomach. The relationship between food insecurity and the increased risk of nonadherence to antiretroviral therapy among HIV infected adults was also reported in the Democratic republic of Congo [38]. In North eastern Uganda eating one meal a day was reported as a risk factor for ART non adherence [39]. The study on the assessment of barriers and facilitators to patient's adherence to ART in Zambia reported that individual beliefs that ART should not be used without adequate food made clients skip ART medication when they did not have enough food to eat [40]. Lack of food was also among the important barriers to ARV adherence in a qualitative study from South Africa [41]. The fear about hunger and food insecurity is also reported to contribute into HIV patient's ART treatment default [42]. These findings underline the need for integrating locally available food in the health education given to those diagnosed with HIV to improve perceived food shortages when taking prescribed medication. 


\section{Conclusion}

This study has revealed several interacting factors which contribute to defaulting from ART. These factors operate at individual, community, health facility and policy levels. There is a need to develop health promotion interventions which address each of these challenges in order to improve compliance with recommended ART management.

\section{References}

[1] UNAIDS., 2015. Understanding -Track: Accelerating Actions to End the AIDS Epidemic by 2030. Accessed on 22 November, 2020 https://www.unaids.org/sites/default/files/media_asset/201506 JC2743_Understanding_FastTrack_en.pdf.

[2] Adeniyi OV, Ajayi AI, Ter Goon D, Owolabi EO, Eboh A, Lambert J. Factors affecting adherence to antiretroviral therapy among pregnant women in the Eastern Cape, South Africa. BMC infectious diseases. 2018; 18 (1): 175.

[3] Chalker J, Andualem T, Minzi O, Ntaganira J, Ojoo A, Waako $\mathrm{P}$, et al. Monitoring adherence and defaulting for antiretroviral therapy in 5 east African countries: an urgent need for standards. Journal of the International Association of Physicians in AIDS Care. 2008; 7 (4): 193-9.

[4] Rachlis B, Ahmad F, van Lettow M, Muula AS, Semba M, Cole DC. Using concept mapping to explore why patients become lost to follow up from an antiretroviral therapy program in the Zomba District of Malawi. BMC health services research. 2013; 13 (1): 1-11.

[5] Van Cutsem G, Ford N, Hildebrand K, Goemaere E, Mathee S, Abrahams M, et al. Correcting for mortality among patients lost to follow up on antiretroviral therapy in South Africa: a cohort analysis. PLoS One. 2011; 6 (2): e14684.

[6] Sahay S, Reddy KS, Dhayarkar S. Optimizing adherence to antiretroviral therapy. The Indian journal of medical research. 2011; 134 (6): 835.

[7] DiMatteo MR. Variations in patients' adherence to medical recommendations: a quantitative review of 50 years of research. Medical care. 2004: 200-9.

[8] Fernandez-Lazaro CI, García-González JM, Adams DP, Fernandez-Lazaro D, Mielgo-Ayuso J, Caballero-Garcia A, et al. Adherence to treatment and related factors among patients with chronic conditions in primary care: a cross-sectional study. BMC family practice. 2019; 20 (1): 132.

[9] Malta M, Magnanini MM, Strathdee SA, Bastos FI. Adherence to antiretroviral therapy among HIV-infected drug users: a meta-analysis. AIDS and Behavior. 2010; 14 (4): 73147.

[10] Achappa B, Madi D, Bhaskaran U, Ramapuram JT, Rao S, Mahalingam S. Adherence to antiretroviral therapy among people living with HIV. North American journal of medical sciences. 2013; 5 (3): 220.

[11] Enane LA, Vreeman RC, Foster C. Retention and adherence: global challenges for the long-term care of adolescents and young adults living with HIV. Current Opinion in HIV and AIDS. 2018; 13 (3): 212-9.
[12] Heestermans T, Browne JL, Aitken SC, Vervoort SC, Klipstein-Grobusch K. Determinants of adherence to antiretroviral therapy among HIV-positive adults in subSaharan Africa: a systematic review. BMJ global health. 2016; 1 (4): e000125.

[13] Global information and education on HIV and AIDS; Accessed on 12th December, 2020 at https://www.avert.org/professionals/hiv-around-world/subsaharanafrica/tanzaniahttps://www.avert.org/professionals/hivaround-world/sub-saharan-africa/tanzania.

[14] Mannheimer S, Friedland G, Matts J, Child C, Chesney M, AIDS TBCPfCRo. The consistency of adherence to antiretroviral therapy predicts biologic outcomes for Human Immunodeficiency Virus - infected persons in clinical trials. Clinical infectious diseases. 2002; 34 (8): 1115-21.

[15] Braun V, Clarke V. What can "thematic analysis" offer health and wellbeing researchers? International journal of qualitative studies on health and well-being. 2014; 9.

[16] Weiser S, Wolfe W, Bangsberg D, Thior I, Gilbert P, Makhema $\mathrm{J}$, et al. Barriers to antiretroviral adherence for patients living with HIV infection and AIDS in Botswana. JAIDS-HAGERSTOWN MD- 2003; 34 (3): 281-8.

[17] Musumari PM, Feldman MD, Techasrivichien T, Wouters E, Ono-Kihara M, Kihara M. "If I have nothing to eat, I get angry and push the pills bottle away from me": A qualitative study of patient determinants of adherence to antiretroviral therapy in the Democratic Republic of Congo. AIDS care. 2013; 25 (10): 1271-7.

[18] Koole O, Denison JA, Menten J, Tsui S, Wabwire-Mangen F, Kwesigabo G, et al. Reasons for missing antiretroviral therapy: results from a multi-country study in Tanzania, Uganda, and Zambia. PloS one. 2016; 11 (1): e0147309.

[19] McGuire M, Munyenyembe T, Szumilin E, Heinzelmann A, Le Paih M, Bouithy N, et al. Vital status of pre-ART and ART patients defaulting from care in rural Malawi. Tropical Medicine \& International Health. 2010; 15: 55-62.

[20] Dessie G, Wagnew F, Mulugeta H, Amare D, Jara D, Leshargie CT, et al. The effect of disclosure on adherence to antiretroviral therapy among adults living with HIV in Ethiopia: a systematic review and meta-analysis. BMC infectious diseases. 2019; 19 (1): 1-8.

[21] Tshweneagae GT, Oss VM, Mgutshini T. Disclosure of HIV status to sexual partners by people living with HIV. curationis. 2015; 38 (1): 1-6.

[22] Yonah G, Fredrick F, Leyna G. HIV serostatus disclosure among people living with HIV/AIDS in Mwanza, Tanzania. AIDS research and therapy. 2014; 11 (1): 1-5.

[23] Forsythe S, Lee B, Tarimo K, Silvan B, Balampama M, Chitty J, Bowsky S. HIV treatment support services in Tanzania: a cost and efficiency analysis at facility and community levels. African Journal of AIDS Research. 2019 Dec 6; 18 (4): 30614.

[24] Chirambo L, Valeta M, Kamanga TMB, Nyondo-Mipando AL. Factors influencing adherence to antiretroviral treatment among adults accessing care from private health facilities in Malawi. BMC public health. 2019; 19 (1): 1-11. 
[25] Byakika-Tusiime J, Polley EC, Oyugi JH, Bangsberg DR. Free HIV antiretroviral therapy enhances adherence among individuals on stable treatment: implications for potential shortfalls in free antiretroviral therapy. PloS one. 2013; 8 (9): e70375.

[26] Jimmy B, Jose J. Patient medication adherence: measures in daily practice. Oman medical journal. 2011; 26 (3): 155.

[27] Dwajani S, Prabhu M, Ranjana G, Sahajananda H. Importance of medication adherence and factors affecting it. IP International Journal of Comprehensive and Advanced Pharmacology. 2020; 3 (2): 69-77.

[28] Addo B, Sencherey S, Babayara MN. Medication noncompliance among patients with chronic diseases attending a primary health facility in a Periurban district in Ghana. International journal of chronic diseases. 2018; 2018.

[29] Okoronkwo I, Okeke U, Chinweuba A, Iheanacho P. Nonadherence factors and sociodemographic characteristics of HIV-infected adults receiving antiretroviral therapy in Nnamdi Azikiwe University Teaching Hospital, Nnewi, Nigeria. International Scholarly Research Notices. 2013; 2013.

[30] Azia IN, Mukumbang FC, Van Wyk B. Barriers to adherence to antiretroviral treatment in a regional hospital in Vredenburg, Western Cape, South Africa. Southern African Journal of HIV Medicine. 2016; 17 (1).

[31] Watt MH, Maman S, Earp JA, Eng E, Setel PW, Golin CE, et al. "It's all the time in my mind": Facilitators of adherence to antiretroviral therapy in a Tanzanian setting. Social science \& medicine. 2009; 68 (10): 1793-800.

[32] Mills E, Cooper C, Seely D, Kanfer I. African herbal medicines in the treatment of HIV: Hypoxis and Sutherlandia. An overview of evidence and pharmacology. Nutrition journal. 2005; 4 (1): 1-6.

[33] Babb D, Pemba L, Seatlanyane P, Charalambous S, Churchyard G, Grant A, editors. Use of traditional medicine in the era of antiretroviral therapy: experience from South Africa. Proceedings of the 15th International AIDS Conference; 2004.
[34] Homsy J, King R, Balaba D, Kabatesi D. Traditional health practitioners are key to scaling up comprehensive care for HIV/AIDS in sub-Saharan Africa. Aids. 2004; 18 (12): 1723-5.

[35] Endale Gurmu A, Teni FS, Tadesse WT. Pattern of traditional medicine utilization among HIV/AIDS patients on antiretroviral therapy at a university hospital in northwestern Ethiopia: a cross-sectional study. Evidence-based Complementary and Alternative Medicine. 2017; 2017.

[36] Gall A, Shenkute Z, Kiefer D, Jackson J. Ethiopian traditional and herbal medications and their interactions with conventional drugs. Ethno Med. 2009; 1: 1-4.

[37] Young S, Wheeler AC, McCoy SI, Weiser SD. A review of the role of food insecurity in adherence to care and treatment among adult and pediatric populations living with HIV and AIDS. AIDS and Behavior. 2014; 18 (5): 505-15.

[38] Musumari PM, Wouters E, Kayembe PK, Nzita MK, Mbikayi SM, Suguimoto SP, et al. Food insecurity is associated with increased risk of non-adherence to antiretroviral therapy among HIV-infected adults in the Democratic Republic of Congo: a cross-sectional study. PloS one. 2014; 9 (1): e85327.

[39] Olupot-Olupot P, Katawera A, Cooper C, Small W, Anema A, Mills E. Adherence to antiretroviral therapy among a conflictaffected population in Northeastern Uganda: a qualitative study. Aids. 2008; 22 (14): 1882-4.

[40] Sanjobo N, Frich JC, Fretheim A. Barriers and facilitators to patients 'adherence to antiretroviral treatment in Zambia: a qualitative study. SAHARA-J: Journal of Social Aspects of HIV/AIDS. 2008; 5 (3): 136-43.

[41] Nachega JB, Knowlton AR, Deluca A, Schoeman JH, Watkinson L, Efron A, et al. Treatment supporter to improve adherence to antiretroviral therapy in HIV-infected South African adults: a qualitative study. JAIDS Journal of Acquired Immune Deficiency Syndromes. 2006; 43: S127-S33.

[42] Weiser SD, Tuller DM, Frongillo EA, Senkungu J, Mukiibi N, Bangsberg DR. Food insecurity as a barrier to sustained antiretroviral therapy adherence in Uganda. PloS one. 2010; 5 (4): e10340. 